\title{
Disruption of gamma-delta relationship related to working memory deficits in first-episode psychosis
}

\author{
Pascal Missonnier $^{1,2} \cdot$ Anne Prévot $^{1,3} \cdot$ François R. Herrmann $^{4} \cdot$ Joseph Ventura $^{5} \cdot$ Anna Padée $^{1} \cdot$ Marco C. G. Merlo $^{1}$
}

Received: 14 November 2018 / Accepted: 14 December 2019 / Published online: 19 December 2019

(c) Springer-Verlag GmbH Austria, part of Springer Nature 2019

\begin{abstract}
Working memory (WM) deficits constitute a core symptom of schizophrenia. Inadequacy of WM maintenance in schizophrenia has been reported to reflect abnormalities in the excitation/inhibition (E/I) balance between pyramidal neurons and parvalbumin basket cells, which may explain alterations of the dynamics of gamma and delta oscillations. To address this issue, we assessed event-related gamma $(35-45 \mathrm{~Hz})$ and delta $(0.5-4 \mathrm{~Hz})$ oscillatory responses in a visual $n$-back WM task in patients with first-episode psychosis (FEP) and healthy controls (HC). Periodicity analyses of oscillations were computed to explore the relationship between the psychiatric status and the WM load-related processes reflected by each frequency range. The correspondence between nested delta-gamma oscillations was estimated to assess the strength of the frontal E/I balance. In HC, gamma oscillations were synchronized by the stimulus in a 50-150 ms time range for all tasks, and periodicity of the delta cycle was comparable between the tasks. In addition, synchronization of gamma oscillations in HC occurred at the maximal descending phase of the delta cycle half-period, supporting the coexistence of delta-nested gamma oscillations. Compared with controls, FEP patients showed a lack of gamma synchronization independently of the nature of the task, and the period of delta oscillation increased significantly with the difficulty of the WM task. We thus demonstrated in FEP an inability to encode multiple items in short-term memory associated with abnormalities in the relationship between oscillations related to the difficulty of the WM task. These results argue in favor of a dysfunction of the E/I balance in psychosis.
\end{abstract}

Keywords First-episode psychosis · Delta and gamma nesting · Working memory · Dorsolateral prefrontal cortex · Excitation/inhibition balance

Pascal Missonnier

pascal.missonnier@unifr.ch

1 Laboratory for Psychiatric Neuroscience and Psychotherapy, Department of Neuroscience and Movement Sciences, Medicine Section, Faculty of Science and Medicine, University of Fribourg, Chemin du Musée 5, 1700 Fribourg, Switzerland

2 Mental Health Network Fribourg (RFSM), Sector of Psychiatry and Psychotherapy for Adults, L'Hôpital 140, Case postale 90, 1633 Marsens, Switzerland

3 School of Health Sciences (HEdS-FR), University of Applied Sciences and Arts Western Switzerland, 1700 Fribourg, Switzerland

4 Division of Geriatrics, Department of Rehabilitation and Geriatrics, Geneva University Hospitals and University of Geneva, 1226 Thônex, Switzerland

5 Department of Psychiatry and Biobehavioural Sciences, University of California, Los Angeles (UCLA), Los Angeles, CA, USA

\section{Introduction}

Schizophrenia is a severe psychiatric disorder with a lifetime risk of about 1\% (American Psychiatric Association 2013). Delusions, hallucinations, and disorganization of thought, as well as impairments of social functioning, characterize the disease (American Psychiatric Association 2013). In addition, these clinical symptoms are associated with important cognitive dysfunctions which appear to result from alterations of neuronal oscillations activity (Uhlhaas and Singer 2010), thereby impairing communication and coordination between different brain regions. This is important because synchronous activities of neuronal networks play a key role in the maturation of the brain (Uhlhaas et al. 2010) and a default during a late period of brain development is another well-known clinical characteristic of schizophrenia (Lisman 2012).

Numerous findings suggest that a disruption of the balance of the cortical ratio between excitation and inhibition 
(E/I) in the dorsolateral prefrontal cortex (DLPFC), a critical liability region in schizophrenia, could be the origin of cognitive deficits (Anticevic and Lisman 2017).

Typically, the $\mathrm{E} / \mathrm{I}$ balance is ensured by a local synergistic relation between: (1) excitatory glutamatergic pyramidal neurons oscillating in the delta low-frequency band (0.5-4 Hz) (Pratt et al. 2017); and (2) parvalbumin basket cells (PVBCs), a type of interneuron from the GABAergic neurons class extensively connected with several pyramidal neurons, thereby constituting neuronal networks that synchronize pyramidal cell firing (for review, Fries et al. 2007; Buzsáki and Wang 2012). PVBCs play an important role in the generation of high-frequency gamma oscillations $(>30 \mathrm{~Hz}$ ), due to their fast-spiking characteristics and the synaptic short-time dynamics (Zaitsev and Lewis 2013).

In schizophrenia, however, these gamma oscillations suffer a decrease of the intensity of their signal (for review, Reilly et al. 2018). This results first from multiple pre- and postsynaptic receptor abnormalities in PVBCs (Lewis et al. 2011; Lewis 2012). Moreover, the synthesis as well as the release of GABA by PVBCs decreased, leading to a lower chloride influx into pyramidal cells, hence a reduction of the hyperpolarizing role of GABA, as well as a weakened pyramidal cell inhibition (Lewis et al. 2011). Taken together, these observations have suggested a new model of E/I dysfunction in schizophrenia in which the decrease of PVBCs inhibition would reflect a compensation for an upstream deficit in pyramidal cell excitation. As a result, in this model, the cortical $\mathrm{E} / \mathrm{I}$ ratio is set to a lower level of both excitation and inhibition. Therefore, in situations of high levels of cognitive demand, the schizophrenic brain is unable to generate adapted power gamma oscillations, resulting in impaired cognition (Uhlhaas and Singer 2010, 2013).

Working memory (WM) is a high-level cognitive function which refers to temporary online maintenance of new information in short-term memory (STM) to process and use relevant information (Baddeley 2000). In the dorsolateral prefrontal cortex (DLPFC), interactions of neuronal theta $(4-8 \mathrm{~Hz})$ and gamma $(>30 \mathrm{~Hz})$ oscillations are essential for cognitive control, especially of WM (Mainy et al. 2007; Palva et al. 2010; Lisman and Jensen 2013a, b; Kamiński et al. 2011; Lundqvist et al. 2016; Barr et al. 2017). Physiologically, coupled theta and gamma oscillations create a neural code allowing the representation of multiple items in a defined order (Lisman and Buzsáki 2008; Kamiński et al. 2011; Leszczyński et al. 2015; Rajji et al. 2017). Multiple bits of information are thus simultaneously maintained in the WM by consecutive gamma oscillations (cycles of a period of $25 \mathrm{~ms}$ ), while lower-frequency oscillation bands underlie the sequential order of the information. Moreover, delta phase $(0.5-4 \mathrm{~Hz})$ modulates the amplitude of theta, so that gamma oscillations (through their linkage with theta oscillations) are locked into the maximal ascending phase of delta (Lisman and Buzsáki 2008; Lisman and Jensen 2013a, b). Rhythmic brain oscillations have also been shown to be related in a nested fashion (Lakatos et al. 2005; Lisman and Jensen 2013a, b). Therefore, delta oscillation serves as a gating mechanism for WM operations. Altogether, WM maintenance of multiple bits of information clearly relies on a hierarchy of neural oscillations.

Working memory deficits constitute a core symptom of schizophrenia. Previous contributions documented that aberrant event-related gamma oscillation parameters occur in schizophrenia during WM tasks (Uhlhaas et al. 2008; Haenschel et al. 2009). This abnormal profile of gamma activity exists in the initial phases of WM processes and during the initial period of encoding, as well as during the maintenance phase (Uhlhaas et al. 2008; Spencer et al. 2004, 2008). This highlights the deficit in psychotic patients for modulating neural networks engaged during WM-related processes in an appropriate manner. Many studies have reported similar neurophysiological disturbances in gamma oscillation patterns in first-episode psychosis (FEP) patients (Lett et al. 2014; Senkowski and Gallinat 2015; Başar-Eroglu et al. 2007; Reilly et al. 2018). Moreover, dysfunctional oscillations in magnetoencephalography delta $(8-12 \mathrm{~Hz})$ frequency ranges are present during the engagement of different processes of WM in schizophrenia (Ince et al. 2009). Interestingly, previous findings in an auditory modality documented WM deficits associated with a major delta desynchronization in EEG, as well as a moderate impairment of gamma synchronization (Puvvada et al. 2018). Other contributions pointed to deteriorated evoked (as compared to induced) delta responses to target stimuli in schizophrenia patients (Doege et al. 2010; Güntekin and Başar 2016), supporting the idea of deficits in the initial phases of WM processes. These findings strongly suggest that deficits in delta generation in patients would exert a more fundamental role and stronger contributions to WM deficits.

Until now, coordination between oscillations in psychosis was only studied between theta phase and gamma amplitude (Barr et al. 2017). The interaction between delta and gamma oscillations has not yet been investigated in early psychosis, although polyrhythmic working is the critical issue for an adequate comprehension of the neuronal networks activity. In this context, analysis of the time relationship of high- and low-frequency oscillations in a WM paradigm seems particularly adapted to address the hypothesis that a disrupted frontal $\mathrm{E} / \mathrm{I}$ balance may induce or underlie impairments in DLPFC-dependent behaviors.

First, we performed an EEG activation study in FEP patients and healthy controls (HC). We then analyzed the synchronization of oscillations and the relationship between frequencies of delta and gamma oscillations in participants that successfully performed an $n$-back WM task, as well as oddball detection and $n$-back passive fixation tasks. 
As postulated by the model of cortical dysfunction, we hypothesized that the more difficult the task, the stronger the decrease of oscillations periodicity would be in FEP patients. Using analysis based on the timing sequence of cycles of oscillations, we were able to unveil the presence and the time of occurrence of a synchronicity between delta and gamma oscillations.

\section{Methods}

\section{Participants}

The present work is based on data collected for a previous study performed in a cohort of healthy controls and firstepisode psychosis patients (Missonnier et al. 2012, 2017). The protocol was approved by the Ethics Committee of the University Hospitals of Geneva, Switzerland. Details of data collection and population demographics have been extensively described previously (Missonnier et al. 2012, 2017).

First, eighteen adult healthy controls (HC) and fifteen patients with first-episode psychosis (FEP) were recruited in the specialized program for young adult psychiatric patients (JADE) in Geneva. As the two groups differed in age (Student's $t$ test, $t=3.501$; degrees of freedom $(d f)=31$, $p=0.0014$ ) but not by gender (Pearson $\chi^{2}(1)=1.8658$, $p=0.172$ ), we performed a manual age matching by discarding the two oldest healthy controls and the younger patient, resulting in a new $\mathrm{HC}$ group $(N=16$ unique controls) and a new FEP group ( $N=14$ unique patients). This process decreased the group age difference to 2.14 instead of 2.60 years (Student's $t$ test, $t=2.9856 ; d f=28, p=0.0058$ ), while gender proportion remains equivalent (Pearson $\chi^{2}$ $(1)=1.4286, p=0.232)$. Using a mathematical procedure of matching (see method below) the two groups were made numerically equivalent and do not differ either on gender $($ McNemar's chi square, odds ratio $=1.000)$, or on age $(\operatorname{Pr}(|T|>|t|)=0.0728)$. Two experienced independent psychiatrists made the diagnosis according to the DSM-IV criteria (American Psychiatric Association 1994). Six patients were diagnosed with schizophreniform psychotic disorder and eight with schizophrenia. Five patients had a history of recreational cannabis use. Patients with other types of drug abuse, as well as with history of sustained head injury or other neurologic or psychiatric disorders, were excluded. The severity of the patients' symptoms was rated using items of the Brief Psychiatric Rating Scale (BPRS) (Ventura et al. 1993; Missonnier et al. 2012, 2017).

Controls had no history of sustained head injury or other neurologic or psychiatric disorders. All participants had normal or corrected-to-normal vision. Demographic characteristics of all participants and clinical information of FEP patients are provided (Table 1).
Table 1 Demographic and clinical characteristics of the healthy controls (HC) and first-episode psychosis (FEP) patients

\begin{tabular}{ll}
\hline Healthy controls (HC) & \\
Age, years & $24.7 \pm 1.4$ \\
Gender (f/m) & $8 / 8$ \\
First-episode psychosis (FEP) & $22.5 \pm 2.3$ \\
Age, years & $4 / 10$ \\
Gender (f/m) & \\
Positive symptoms (BPRS items) & $1.6 \pm 0.9$ \\
Bizarre behavior & $2.5 \pm 1.3$ \\
Unusual thought content & $1.7 \pm 1.4$ \\
Hallucination & $1.7 \pm 1.4$ \\
Suspiciousness & \\
Negative symptoms (BPRS) & $3.2 \pm 1.2$ \\
Blunted effect & $1.9 \pm 1.5$ \\
Emotional withdrawal & \\
\hline
\end{tabular}

Data represent means $\pm \mathrm{SD}$

$B P R S$ Brief Psychiatric Rating Scale

\section{Experimental design}

Participants performed a $n$-back working memory task, in which subjects viewed a continuous stream of stimuli (pseudo-random sequences of letters) and had to press a button for each stimulus whenever it matched the stimulus shown in stimuli earlier in the sequence ( $n=1$ - or 2-back) (Nystrom et al. 2000). The protocol also included an attentional active detection task (memory free) and a passive fixation of letter series task (see Fig. 1 and Missonnier et al. 2012, 2017 for thorough description of the experimental procedures). The duration of the experiment was approximately $40 \mathrm{~min}$.

\section{Electrophysiological recordings}

Continuous EEG (Brain Quick system 98, Micromed, Treviso, Italy) was recorded using 20 surface electrodes placed over the scalp according to the 10-20 international electrode placement system (Homan et al. 1987), with linked earlobes as a reference. Skin impedance was kept below $5 \mathrm{k} \Omega$. Electrophysiological signals were sampled at $1024 \mathrm{~Hz}$ with a lower and upper cutoff of $0.33 \mathrm{~Hz}$ and $120 \mathrm{~Hz}$, respectively (DC amplifiers, Micromed). The electrooculogram was recorded using two pairs of bipolar electrodes in both vertical and horizontal directions. Single transistor-transistor logic (TTL) pulses synchronized with stimulus onset were recorded and used offline to segment the continuous EEG data into epochs time locked to stimulus onset. 

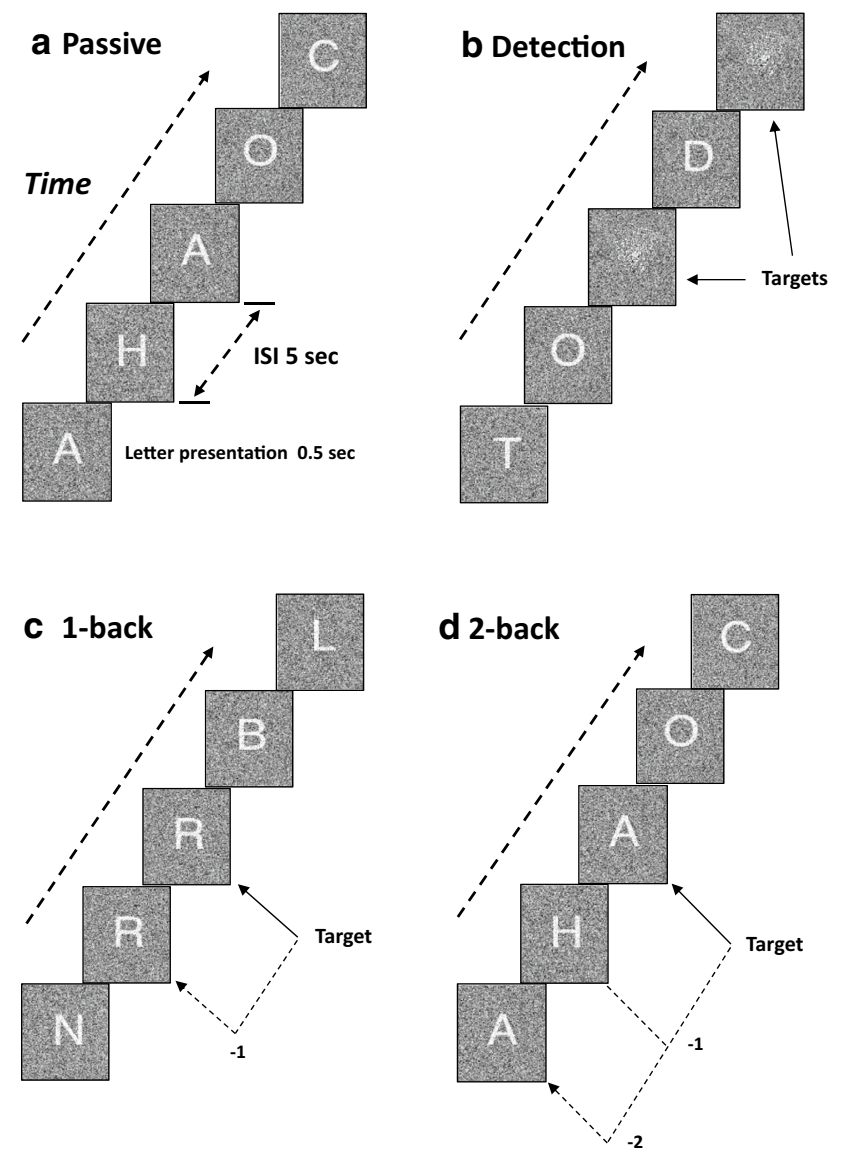

Fig. 1 Schematic representation of the four experimental tasks. a In the passive fixation task, letter series identical to the 2-back working memory task (d) are presented. The subject must only watch the series; no motor action is required. b In the detection attention task, the subject must press a pushbutton as soon as a background patch without letter is presented (Target). c, d In the 1-back and 2-back working memory tasks, the subject must press a button when the letter presented (Target) is identified as being identical to the one presented one trial back (i.e., 1-back task), or two trials back (i.e., 2-back task). Stimulus duration $=$ letter presentation $=0.5 \mathrm{~s}$; inter-stimulus interval $($ ISI $)=5 \mathrm{~s}$

\section{Data processing}

Data were analyzed with the BrainVision Analyzer 2 software (Brain Products GmbH, Munich, Germany). After artifact removal and offline correction of ocular artifacts with an independent component analysis using the infomax algorithm (Jung et al. 2000), the spatial resolution of the EEG data was enhanced by surface Laplacian estimation (regularized 3D spline function, order 4). This acts as a high-pass spatial filter reducing head volume conduction and cancels out the influence of the reference electrode (Perrin et al. 1987; Babiloni et al. 1996). All subsequent analyses were performed on the Laplacian-transformed EEG signal $\left(\mu \mathrm{V} / \mathrm{m}^{2}\right)$ computed at each electrode. Next, the Laplaciantransformed EEGs detached into windows of $4000 \mathrm{~ms}$ (starting $500 \mathrm{~ms}$ before stimulus onset) were automatically scanned for contamination by muscular or electrode artifacts (criteria for rejection: voltage step $>50 \mu \mathrm{V} / \mathrm{ms}$ or peakto-peak deflection within 300-ms intervals $>200 \mu \mathrm{V} / \mathrm{ms}$ ). The remaining trials were inspected visually to control for residual minor artifacts. To eliminate effects from manual responses and exclude the confounding effect of motor processing, data from trials with correct answers without motor responses were averaged according to task conditions. Then, event-related oscillations were averaged over a window of $1050 \mathrm{~ms}$ with a $525-\mathrm{ms}$ prestimulus onset, and filtered at $0.5-4 \mathrm{~Hz}$ (delta) and 35-45 Hz (gamma) frequency bands using a narrow band-pass filter ( $-48 \mathrm{~dB} /$ octave). Analyses of the segment-evoked oscillations included the frontal (F3, Fz, F4) electrodes. Finally, to exclude task-related effects on additional oscillatory components, we also investigated event-related oscillations on theta $(4.5-7.5 \mathrm{~Hz})$ and alpha (8-12 Hz) frequency bands.

On average for healthy participants, the number of artifact-free trials (epochs) was 58.50 $\pm 10.83,61.31 \pm 8.01$, $57.25 \pm 16.94$ and $58.00 \pm 9.39$ in passive and detection tasks, as well as 1- and 2-back tasks, respectively. Similar numbers were obtained for FEP with $57.56 \pm 16.16$, $48.06 \pm 17.81,52.19 \pm 17.60$ and $51.81 \pm 17.71$, respectively.

\section{Estimation of synchronization of event-related oscillations and statistical analysis}

\section{Event-related spectrograms analysis}

To ensure the accuracy of the frequency bands and time periods retained in our analyses, we performed a short-time fast transform Fourier (STFFT) across time and frequence (i.e., STFFT spectrogram) analysis. The event-related SFFT spectrograms were assessed over a window of $5000 \mathrm{~ms}$ including a $2000 \mathrm{~ms}$ prestimulus using custom scripts under MATLAB v7.04 (Mathworks, Natick, MA), with addition of the Chronux software package (chronux.org). Data from all participants in both groups were aggregated for each task separately. We also aggregated data of 1-back and 2-back tasks together. Results will be presented for electrodes F3, $\mathrm{F} 4, \mathrm{Fz}$, as well as the average of all three electrodes (see Fig. 3 in "Results").

Finally, we performed a baseline correction on each spectrogram by averaging the power density for each frequency over the first $2000 \mathrm{~ms}$ prestimulus, hence subtracting the resting state activity occurring before time zero of the experiment from further measurements.

Trial by trial event-related spectral energy changes were analyzed from 0.3 to $60 \mathrm{~Hz}$ within one analysis window. This method allows for visualizing significant variations of the energy spectrum (power $[\mathrm{dB}]$ ) during the time of the frequencies of interest with the voltage-in-time average. We 
chose a cold-to-hot color rendering, with green/blue denoting the lowest energy and orange/red the highest energy.

\section{Synchronicity analysis}

First, amplitudes of oscillations were plotted against time, allowing for a time series analysis. In the present context, this method offers a tool to formalize the periodicity of a signal (i.e., a complete cycle of an oscillation, or signal period) which corresponds to analysis of oscillations synchronicity. Thus, the time series analysis allows to determine precisely whether neural populations are simultaneously activated, resulting in a cycle of oscillation (Buzsáki and Wang 2012).

The estimation of the period synchronicity consisted of first identifying automatically the points on the $x$-axis (time) where the oscillation changes sign (i.e., period shift), also called nodes. The nodes values were computed from a simple linear regression formula expressed as the minus of the intercept value divided by the slope of the curve passing between the points before and after the crossing of the $x$-axis. Then, distances between two adjacent nodes were calculated to obtain the half-period of the oscillation. Finally, by dividing the standard deviation of the half-period by its mean, we obtained the coefficient of variation of the half-period. The phase synchronicity measurement was performed for each subject and task for both gamma and delta oscillations.

For gamma oscillations, we analyzed the half-period coefficient of variation between 50 and 150 ms using repeatedmeasures ANOVA with group (HC, FEP) and task (passive, detection, 1-back, 2-back) effects as well as their interactions, with the task being the repeated factor.

For delta oscillations, we analyzed the first two halfperiods with repeated-measures linear regression models to compare the average period (i.e., the dependent variable) between both groups while adjusting for task, the average value of the first node, with a group by task interaction.

A matching procedure exploring potential effects of independent factors was performed, with age and gender as matching variables. First, to ensure that both groups were comparable despite an uneven number of patients, and therefore that the matching procedure was adequate, we performed two additional tests, namely the McNemar's Chi-squared test as well as paired $t$ test. The exact McNemar's Chi square significance probability was $=1.0000$, proving that both groups were strictly identical for gender. Likewise, the paired $t$ test value was $\operatorname{Pr}(|T|>|t|)=0.0728$, showing that both groups were also comparable for age $(\mathrm{HC}=24.7 \pm 1.4, \mathrm{FEP}=24.1 \pm 1.17)$. We were therefore able to compare the first two half-periods of the delta cycle between controls and gender- and age-matched FEP groups: each case was matched to one control for gender and age using Stata's "nnmatch" command. This function provides nearest-neighbor matching estimators for average treatment effects (statistic average treatment effects; SATC), thus allowing individual observations to be used as a match more than once. Compared with matching without replacement, this method may increase the level of variance but foremost lowers bias (Abadie et al. 2004).

\section{Behavioral statistics}

Reaction time and performance measures were normally distributed according to the Shapiro-Francia W test. Group (controls vs. first-episode patients), task condition (passive, detection, 1-back, 2-back), and a group by task interaction term were included as independent variables in a repeatedmeasures analysis of variance (ANOVA) to analyze their respective influence on each of the dependent variables (reaction time, performance measures and number of EEG epochs). The significance values of the repeated factors in the ANOVA were determined using Box's conservative correction factor. We performed post hoc analysis using the Scheffé test.

The statistical threshold was set at $p<0.05$. Statistical analyses were performed using the Stata software package, version 15.1 (Statcorp, College Station, TX, USA, 2018).

\section{Results}

\section{Behavioral data}

During the n-back testing, reaction times increased as the tasks became more difficult in both HC and FEP $(F(2$, $56)=23.16, p<0.001)$ demonstrating a task effect. Post hoc Scheffé test revealed that the 2-back reaction times were significantly increased as compared to detection and 1-back (Scheffé $p<0.001$ ). Neither group effect nor group and task interaction were observed (Fig. 2a).

All participants performed well in n-back and detection tasks, with a minimum of $92 \%$ of correct responses (Fig. 2b). As expected, performance was related to task difficulty $(F(2$, $56)=34.36, p<0.001)$ in both groups. Performances for the control and 1-back tasks did not differ significantly, however, all participants performed significantly worse in the 2-back task (Scheffé $p<0.001$ ) demonstrating a task effect.

Participants' performances were also affected by the psychiatric status $(F(1,28)=9.10, p<0.005)$. Post hoc analyses revealed that this effect was restricted to the 2-back task (Scheffé $p<0.001$ ).

\section{Electrophysiological data}

\section{Event-related spectrogram analysis}

The presentation of energy averaged across single trials over frontal electrodes showed a transient energy in delta 


\section{a Reaction Time}

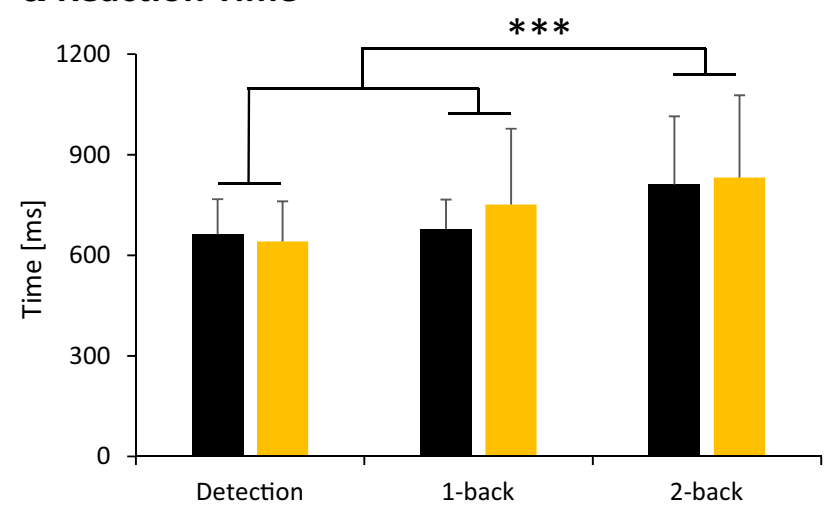

\section{b Performance}

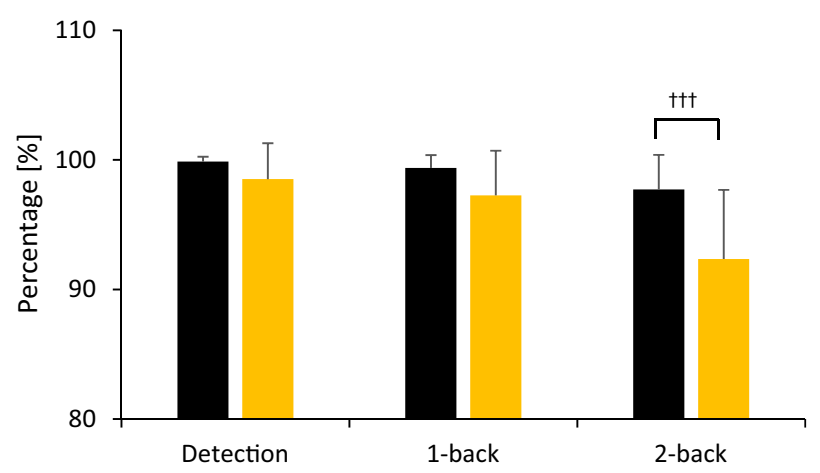

Fig. 2 Bar histograms of reaction time and performance. Averaged reaction times (a) and performances (b) of healthy controls (in black) and first-episode patients (in yellow) for detection and the two n-back tasks. Data are mean \pm SD for each task condition. $* * * p<0.001$ task effect, 2-back vs. detection and 1-back. $\mathrm{H}_{p<0.001 \text { compared with }}$ controls

$(0.5-3.5 \mathrm{~Hz})$ and gamma $(35-45 \mathrm{~Hz})$ frequency band during $0-1000 \mathrm{~ms}$ and $50-250 \mathrm{~ms}$, respectively, after stimulus onset in both groups for no-WM and WM tasks (Fig. 3a, b). These visual observations validate the frequency bands and time windows for subsequent synchronization measurement. No topological differences between hemisphere on brain oscillations was observed (Fig. 3c, for example in the 2-back task for HC), supporting the use of average frontal electrodes.

\section{Synchronization measurement of event-related oscillations}

The time series representation of oscillations was performed to investigate whether synchronization of neuronal activation differed between controls and FEP.

For both groups, the stimulus elicited a series of gamma oscillations in the time range of 0-300 ms. During the interval 50-150 ms post-stimulus (gray area on Fig. 4a), a synchronized gamma activity containing four oscillation cycles of $\sim 25 \mathrm{~ms}$ was observed for all tasks in HC (Fig. 4a, upper panel). In the same time interval, there was no synchronization of neuronal activation (i.e., absence of cycle of $\sim 25 \mathrm{~ms}$ ) in the FEP patients (Fig. 4a, lower panel), as demonstrated by the significantly different gamma dynamics $(t=2.61, p<0.02$ as compared to HC). No task effect or two-way interactions between factors were observed.

Figure. $4 \mathrm{~b}$ displays the circular phase diagram plots of a frontal gamma cycle with a period of $100 \mathrm{~ms}$. The non-overlapping of the circular shapes between tasks in HC (Fig. 4b upper panel) is a graphical illustration that gamma activity was well synchronized by the stimulus in $\mathrm{HC}$ for all tasks. In contrast, the presence of overlap in FEP patients (Fig. 4b lower panel) indicates a lack of synchronization of neuronal populations whatever the task performed by the patients.

The same analysis was repeated after nearest-neighbor matching for age and gender variables and yielded similar results (passive: $p=0.063$; detection: $p=0.285$; 1-back: $\mathrm{p}=0.612$ and 2-back: $p=0.280$ ). Therefore, age and gender do not modify statistical differences, hence our results.

Time series of delta oscillations in a time range 0-800 ms for each task and group is illustrated in Fig. 5. A cycle of delta oscillation of $\sim 400 \mathrm{~ms}$ was observed after stimulus onset in both groups for all tasks.

The time interval, or period, of the delta cycle was not significantly different between the two groups $(t=-0.45$, $p=0.656$ ). However, significant task effects were observed on the period of the cycle of delta oscillation, revealing an increase of the oscillation period for the 1-back $(t=-2.84$, $p=0.012)$ and the 2-back $(t=-2.58, p=0.020)$ tasks. The two-way group $\times$ task interaction revealed that the increased oscillation period was present in FEP only (for 1-back: $t=2.23, p=0.040$; and for 2-back: $t=2.28$, $p=0.037$ ) (see dotted lines in Fig. 5).

The same analysis was repeated after nearest-neighbor matching for age and gender variables and yielded similar results (passive: $p=0.302$; detection: $p=0.777$ 1-back $p=0.110$ and 2-back: $p=0.674$ ).

As expected, the time series in the theta and alpha frequency ranges revealed a cycle of oscillations in a time range of $0-300 \mathrm{~ms}$ and $\sim 20-200 \mathrm{~ms}$ for each task and group, respectively. The periods of theta and alpha cycles were not modulated by group $(t=-1.17, p=0.259$ for theta; $t=-1.72, p=0.106$ for alpha) or task difficulty (passive vs. detection, 1 -and 2-back tasks: $p=0.156, p=0.302$ and $p=0.932$ for theta; $p=0.785, p=0.840$ and $p=0.566$ for alpha). No two-way interactions between conditions were observed either (passive and group: detection, 1-back and 2-back: $p=0.351, p=0.472$ and $p=0.750$ for theta; $p=0.681, p=0.452$ and $p=0.421$ for alpha). 
A

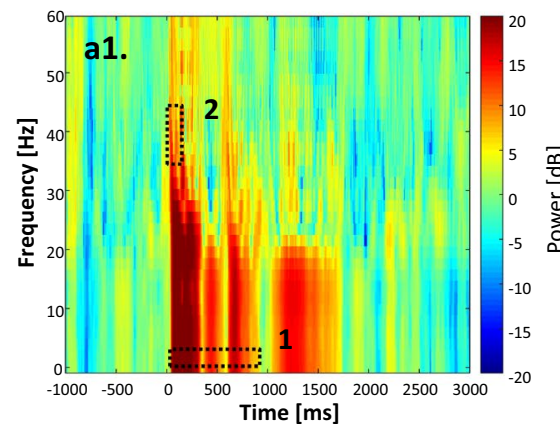

WM HC

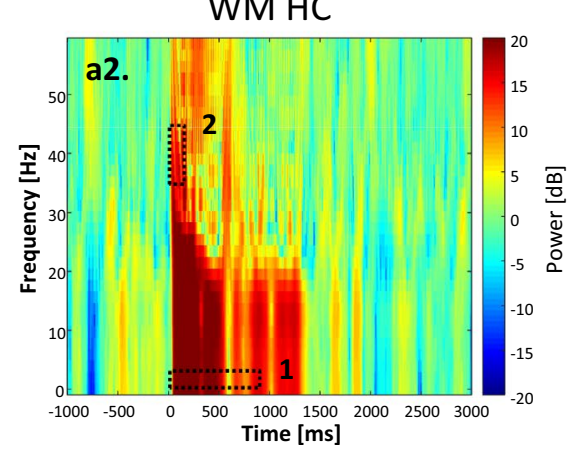

B

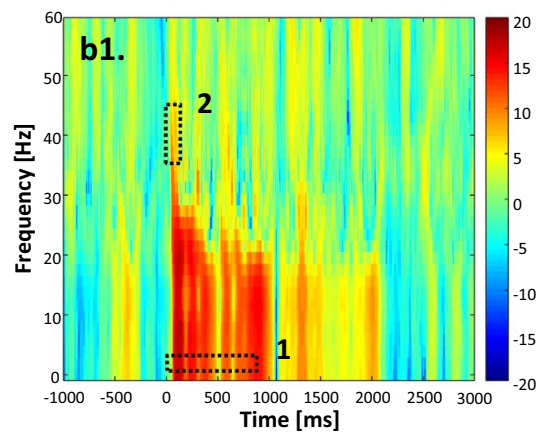

WM FEP

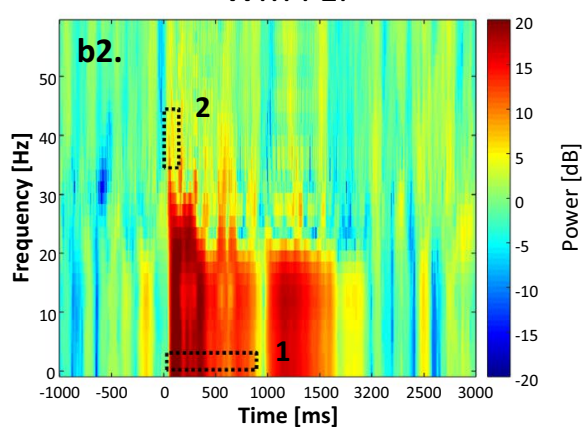

C
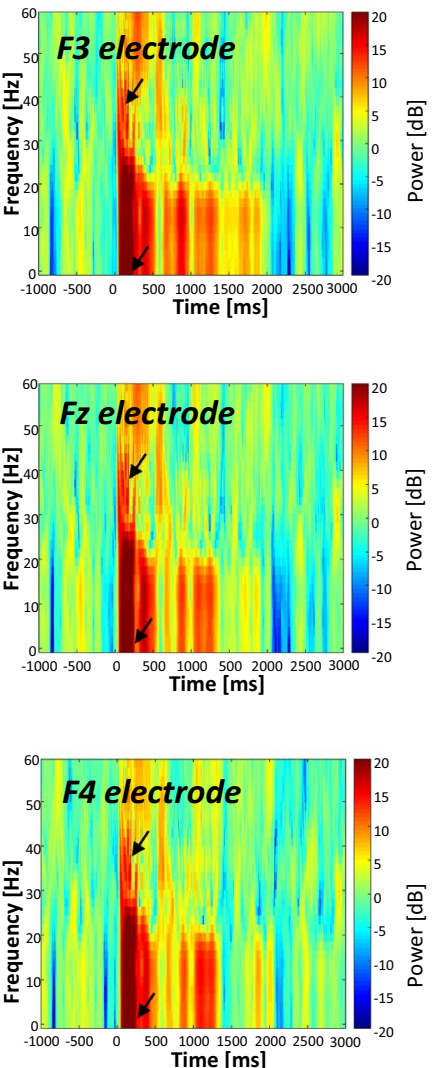

Fig. 3 Grand average frontal spectrograms from short-time fast Fourier transform (STFFT) of EEG. a In healthy controls (HC) during no-working memory (No-WM) (passive and detection combined a1) and working memory (WM) (1- and 2-back combined a2) conditions. b In first-episode psychosis (FEP) during No-WM (passive and detection combined b1) and WM (1- and 2-back combined b2) conditions. For both groups, after stimulus onset a high density (power $[\mathrm{dB}]$ ) of the delta oscillatory activity $(0.5-3.5 \mathrm{~Hz})$ was observed during a sustained time period $(\sim 0-1000 \mathrm{~ms})$, independently of the experimental

\section{Discussion}

We previously reported neurophysiological disturbances in gamma oscillation patterns in first-episode psychosis patients (Missonnier et al. 2017). The present work was designed to further study the nature of these disrupted oscillatory activities in FEP patients. Using a simple visual model to study WM, the current study supports the presence in FEP patients of: (1) a lack of gamma synchronization while performing all tasks; (2) a workload-dependent increase of the delta oscillation period; and (3) a workloaddependent disruption of the synchronization of occurrence of delta/gamma cycles.

Our findings clearly demonstrate that the pathophysiology of WM deficits in FEP can be partly explained by an inability to form the neural coding scheme to encode multiple bits of information in short-term memory via disrupted delta/ condition (dotted square 1). Likewise, a high oscillatory density was observed in the gamma $(35-45 \mathrm{~Hz})$ frequency band (dotted square 2) in a short time period $(\sim 50-150 \mathrm{~ms})$. c Grand average spectrograms of frontal left $(\mathrm{F} 3)$, medial $(\mathrm{Fz})$ and right $(\mathrm{F} 4)$ electrodes for the 2-back in HC. No difference of laterality for delta and gamma oscillations was observed (arrows). The spectrograms are presented with a scale centered on zero in cyan and with deviation from baselines in orange/red. Prestimulus was recorded over $2000 \mathrm{~ms}$ but is presented over $1000 \mathrm{~ms}$ only for easier graphic reading

gamma oscillations. Moreover, we report abnormalities in the relationship between oscillations which are related to the difficulty level of the WM task, arguing in favor of an implication of a dysfunction of the E/I balance in schizophrenia patients.

\section{Gamma synchronization}

Cortical gamma-band activity provides an underlying physiological framework for cognitive operations (Bertrand and Tallon-Baudry 2000; Ward 2011). Notably, this physiological process takes part in encoding the representation of an external object (Lisman and Jensen 2013a, b). To test this concept, our protocol included a sequential presentation of a unique stimulus (i.e. a single letter) for each task, independently of the active or passive nature of the task. The results presented in Fig. 4 demonstrate the presence of synchronous 

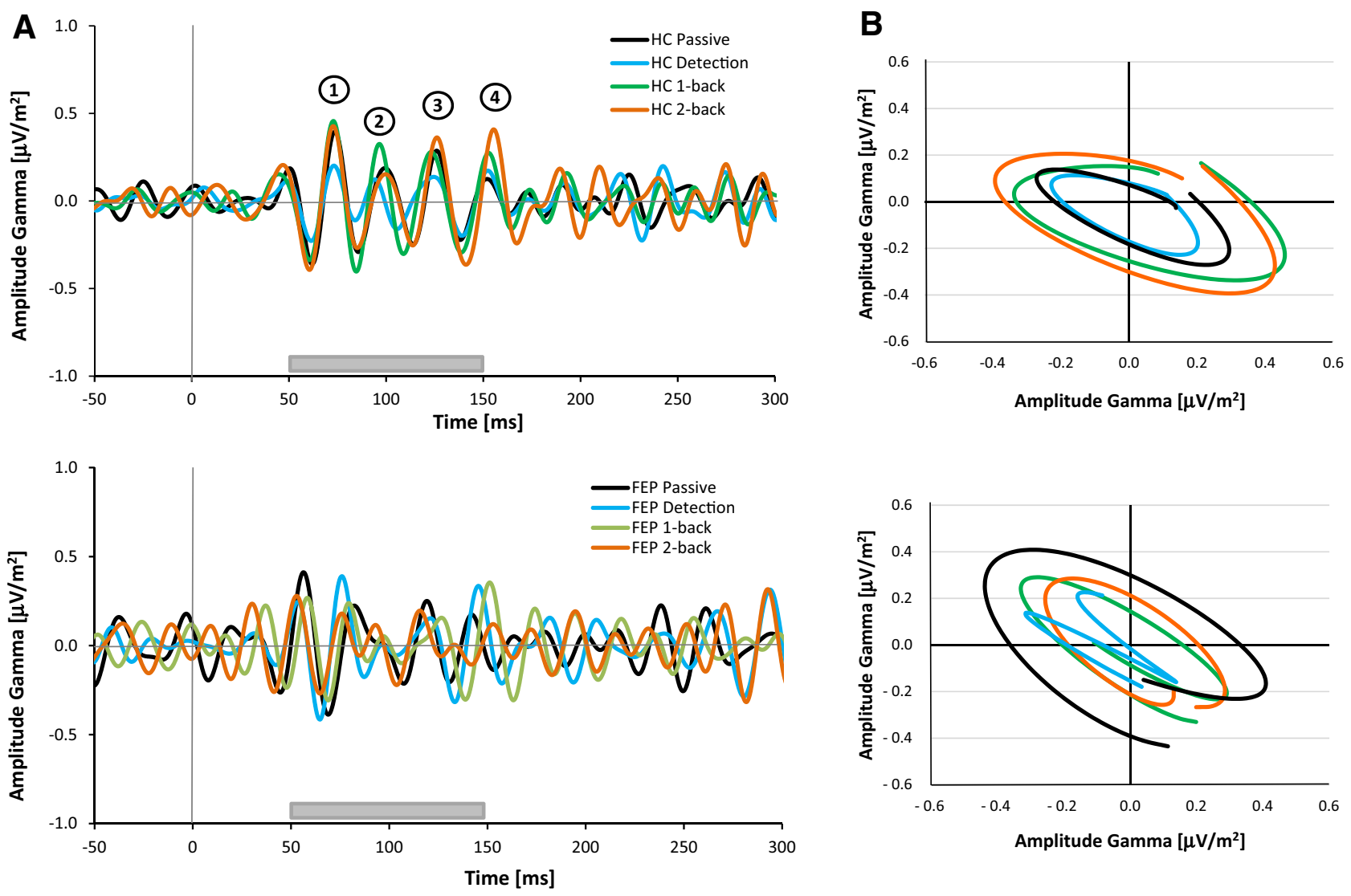

Fig. 4 Grand average frontal gamma oscillations $\left(\mu \mathrm{V} / \mathrm{m}^{2}\right)$ based on groups (upper panels healthy controls $\mathrm{HC}$, lower panels first-episode psychosis FEP) for the four tasks. a As a function of time. The gray area indicates the time interval $(50-150 \mathrm{~ms})$ during which gamma oscillations were synchronized in $\mathrm{HC}$ only (upper panel). Each gamma cycle in this time interval represents one item in short-term memory $(\mathrm{STM})$. Memory span $=4$. No synchronization was found in

gamma oscillations in $\mathrm{HC}$ during $50-150$ ms post-stimulus, but a complete lack of gamma synchronicity in FEP. These changes in gamma activity were neither linked to a motor response, nor task-dependent, but were closely related to stimulus processing, thus suggesting a deficit for mnesic trace formation of a visual stimulus in FEP.

Anatomically and functionally, WM is supported by polyrhythmic interactions between the hippocampus (Barr et al. 2017), the ventral tegmental area and the prefrontal cortex. The phase of delta oscillations facilitates gamma coherence between these structures, so that individual prefrontal gamma cycles play a major role to retain items in memory.

During WM, multiple objects are simultaneously maintained in short-term memory (STM) by a limited number of consecutive gamma cycles and associated with a specific phase range of low-frequency delta oscillations (Lisman and Buzsáki 2008; Roux and Uhlhaas 2014). Precisely, this model suggests that the coding of the first item in a sequence is represented by a gamma oscillation during a first $25-\mathrm{ms}$
FEP patients during the same interval (lower panel). b As a function of average frontal gamma oscillation at time lag-1 (phase diagrams) in the 50-150 ms time interval. The absence of crossover of the ellipses is a marker that oscillations are in phase, which was the case for $\mathrm{HC}$ only (upper panel). The intersection of all ellipses in FEP (lower panel) confirms a lack of gamma synchronization

range that reflects the formation of a transient neuronal assembly (Lisman and Jensen 2013a, b). The second item evokes a second gamma oscillation during the next $25 \mathrm{~ms}$, and so on. Our findings in $\mathrm{HC}$ are in line with this processing for items' maintenance in WM, as we report four synchronous cycles in the $50-150 \mathrm{~ms}$ interval (i.e., four times $25 \mathrm{~ms}$ in a $100 \mathrm{~ms}$ time range) (Fig. 4). Interestingly, the number of cycles was identical whatever the nature of the task and higher than expected for the most difficult WM task, i.e., we could expect three gamma cycles at most for the 2-back condition. These four cycles of gamma oscillations thus represent the number of items stored in STM for our visual paradigm and they correspond to the human memory span, i.e., the limited range of items retained in $\mathrm{STM}=5 \pm 2$ items (Cowan 2001). Therefore, our data support an engagement of transient neuronal assemblies encoding the representation of items in STM in the HC. The present results on gamma oscillations thus describe a normal physiological mechanism of memory encoding. 

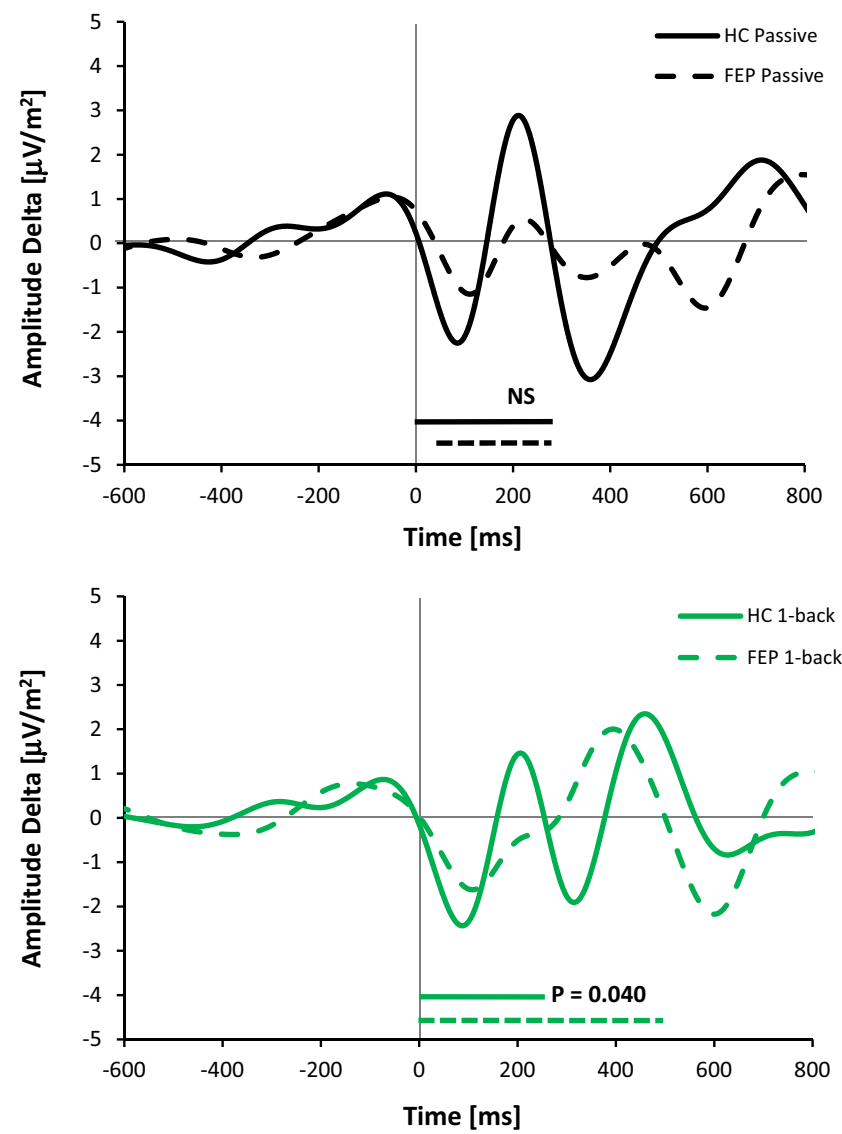

Fig. 5 Grand average frontal delta oscillations $\left(\mu \mathrm{V} / \mathrm{m}^{2}\right)$ as a function of time based on groups for the four tasks. The lines below indicate the period of one cycle of oscillation for both groups (plain lines for

In FEP patients, however, gamma oscillations were not synchronized by stimulus presentation whatever the task, suggesting a lack of formation of cell assemblies coding for individual items to maintain in STM. Supporting this idea, reduced evoked or induced gamma responses were described during WM in patients with schizophrenia (Başar-Eroglu et al. 2007; Başar 2013; Pratt et al. 2017). Despite this lack of cell synchronization, we note that the FEP patients were able to preserve high levels of performance even in the n-back tasks. This fits with our earlier EEG studies in patients with FEP revealing a frontal hyperactivity in lower-frequency theta oscillations combined with an increased parietal activity, as well as an early activation of parietal generators, during both WM and attentional detection tasks (Missonnier et al. 2012). In line with this observation, EEG studies showed oscillations in theta frequency in frontal and posterior brain areas independently of the demand on WM in schizophrenia (Kang et al. 2018). Overall, our results strongly suggest the presence of a fronto-parietal mechanism to compensate for the prefrontal deficiency, which thereby facilitates the
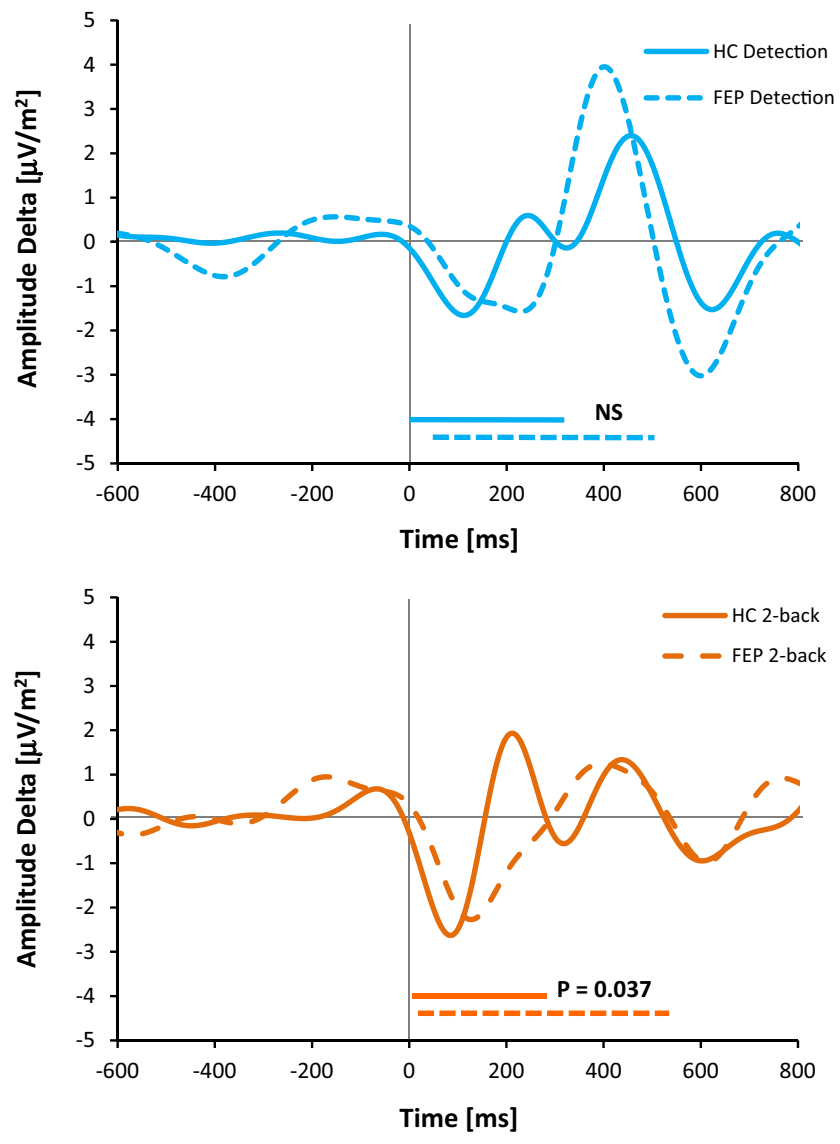

HC, dotted lines for FEP). Note an increase of the period related to the complexity of the tasks in FEP patients only. Statistical significance between groups is indicated on the figure

maintenance of executive performances in FEP patients. In line with the literature, WM deficits might result essentially from impaired synchronicity of prefrontal gamma oscillations (Insel 2010; Gruber et al. 2010).

Physiologically, pyramidal cell firing excites parvalbumin basket cells (PVBCs), which are GABAergic fast-spiking interneurons that in turn inhibit pyramidal cells. When a WM task is initiated in $\mathrm{HC}$, cortical gamma oscillations are generated by a $\sim 25 \mathrm{~ms}$ negative feedback loop in which pyramidal cell firing is increased through pyramidal cell assembly, resulting in an increased excitation of PVBCs, hence an increased inhibition of pyramidal cells. As a result of this negative feedback loop, PVBCs as well as pyramidal cells transiently stop firing (Howard et al. 2003; Schoffelen et al. 2005; Buzsáki and Wang 2012). Once this GABAergic inhibition stops, pyramidal cell firing is reinitiated, thereby generating the next gamma cycle. Functionally, gamma oscillations result from the synchronization of multiple pyramidal cells to fire as a group, which can thereafter be recognized by downstream networks using coincidence detection (Fries 2009). 
In schizophrenia, multiple pre- and postsynaptic abnormalities in PVBCs have been reported, including a reduction in NMDA receptor-mediated synaptic transmission as a result of a major reduction of GAD-67 (i.e., glutamic acid decarboxylase-67), as demonstrated post-mortem in psychotic patients (Lewis 2012). Apparently, a decreased excitation from pyramidal cells would result from a deficit in the number of their dendritic spines. This would in turn lead to a reduction of GAD-67 in NMDA receptors on the PVBCs, hence a decreased inhibition from these PVBCs, thereby modifying the balance of excitation and inhibition and resulting in decreased power of gamma oscillations (Lewis 2012).

Interestingly, findings in animals reported that relatively small changes in the excitatory inputs to fast-spiking interneurons reduce the amplitude of gamma oscillations and lead to a large reduction in the synchronicity of pyramidal cell firing (Buzsáki and Wang 2012). In this context, such a reduction is expected to hinder the triggering of downstream neurons by individual items. Accordingly, no synchronized gamma cycles could be found in our FEP population. Overall, our results demonstrate a neural coding scheme deficit for items' maintenance in WM, associated with impaired prefrontal oscillations in FEP patients. We can strongly hypothesize that this gamma oscillation deficiency was a result of an E/I imbalance, most probably due to dysfunctional PVBCs.

\section{Delta oscillation period}

Delta oscillations were observed in both groups for all tasks. In accordance with the literature (e.g., Leszczyński et al. 2015), the cycle of delta oscillation was phase locked with the stimulus. The period of the slow delta oscillation remained unchanged for all tasks in HC. Yet, in FEP patients the active $n$-back tasks were associated with a longer period, as described in Fig. 5. These results indicate that when the difficulty of the cognitive task increases, the aberrant E/I balance not only affects rapid gamma, but also slow delta oscillations. A recent EEG study in schizophrenic patients demonstrated a stronger relationship between WM performance and major deficits in generating delta oscillations than with gamma oscillations (Puvvada et al. 2018). This suggests that delta deficits may play a more fundamental role than gamma to regulate WM. Our results provide confirmation of the weight of delta phase in neural coding of cognition.

Physiologically, as previously reported for gamma oscillations, NMDA receptors present in PVBCs play a major role in the generation and maintenance of delta oscillations (Steriade 2006; Zhang et al. 2009). A reduction of their activity as seen in schizophrenia would thus lead to a decrease in the amplitude of the delta oscillations as well as an increase of the period, both of which can be seen in our results. Additionally, the properties of delta oscillations are dependent on non-synaptic ion channels, many of which are involved in schizophrenia pathophysiology. Interestingly, a computational modeling study reported that altered expression or properties of schizophrenia-associated voltage-gated calcium channels- and $\mathrm{Ca}^{2+}$ transporters-encoding genes could contribute to impaired dynamics in the delta-frequency band of layer V pyramidal cells (Mäki-Marttunen et al. 2019). From a functional point of view, this neuronal excitability disorder linked to a dysfunction of intracellular calcium handling could alter the response to stimuli in the delta band.

Finally, in schizophrenia, an alteration of the energetic metabolism of neurons has been found (Insel, 2010). Disrupted-in-schizophrenia 1 (DISC1), a candidate gene linked to the disease, has been demonstrated to deregulate mitochondrial function, including oxidative phosphorylation (Park et al. 2010; Prochiantz 2014). PVBCs as well as pyramidal cells require a high-energy intake. An energetic dysfunction in schizophrenic patients would prevent an increase of the neuronal function in case of elevated cognitive demand. Our results showing a lengthening of the periodicity of delta oscillation related to the difficulty of the task in FEP patients are in line with the inability to generate enough excitatory and inhibitory energy to insure the generation of sufficient levels of oscillation.

\section{Combined gamma/delta oscillations}

Brain function is associated with a superposition as well as a synergy of oscillations in the various frequency ranges (Başar 2013). The phase of delta oscillations modulates the amplitude of theta oscillations (Lisman and Jensen 2013a, b). In turn, the phase of theta oscillations modulates the amplitude of gamma oscillations. As a result, the lower frequency delta phase modulates both higher frequency theta and gamma amplitudes in a mechanism referred as nesting (for review, see Hunt et al. 2017). Therefore, investigating the relationship between delta and gamma synchronization rather than individually might uncover additional information.

In $\mathrm{HC}$, synchronization of gamma oscillations in consecutive gamma cycles occurred in the first half-period of the delta cycle for the passive as well as the active tasks. The functioning of WM suggests a combination between periods of rapid and slow-frequency oscillations, where the period of the gamma-band activity that codes items is locked to the phase of the delta oscillation, serving as a gating mechanism. In our study, the occurrence of gamma oscillations at the beginning of the delta cycle strongly supports a loadindependent gamma-delta cross-frequency relationship.

Theoretically, the cortical dysfunction model in schizophrenia implies that, when a cognitive task is performed, 
a decrease of inhibition, hence a weaker power of gamma oscillations, is intended to restore the $\mathrm{E} / \mathrm{I}$ balance in the DLPFC, yet at a lower level (Lewis 2012). This hypothesis assumes that PVBCs cannot restore the functional activity of pyramidal cells for a demanding workload. Thus, abnormalities in E/I balance parameters should lead to disturbances of delta- and gamma-band interactions with deficits for the n-back tasks only for delta but task-independent for gamma oscillations. This pattern of interaction was observed in FEP patients, strongly suggesting that the schizophrenic brain retained the ability to try and readjust the E/I balance, and thereby indicating an insufficient ability to compensate for high levels of cognitive control. Together, our results strongly suggest that the existence of an increase in task difficulty in FEP patients will not only hinder gamma oscillations, but also delta oscillations, as well as their relationship. Psychotic patients will thus suffer early on from inadequate nesting resulting from a limited synaptic plasticity that would not allow neurons to adapt their function adequately in response to increased levels of cognition. This is confirmed by the increase of their reaction times, as well as the significantly decreased performance as compared to HC. An inability of the neural network to adapt their excitatory and inhibitory responses may explain the increased severity of cognitive deficits with task difficulty (Lewis 2012).

\section{Conclusions}

We found a lack of gamma synchronization whatever the task in FEP patients, associated with an increase of the delta oscillation period, both related to the difficulty of the task. These findings suggest that FEP patients are not able to encode multiple items in STM and/or to mobilize sufficient energy to generate oscillations at a sufficient level when the difficulty of the task increases.

The task-dependent abnormalities of frequency relationship argue in favor of a dysfunction of the E/I balance in psychosis. Future studies allowing EEG-functional near-infrared spectroscopy (NIRS)-based assessment of neurovascular coupling would help elucidate whether delta-gamma relationship deficits are accompanied by subtle metabolic changes within the DLPFC.

Funding No funding was received for this work.

\section{Compliance with ethical standards}

Conflict of interest The authors have no actual or potential conflicts of interest.

\section{References}

Abadie A, Drukker D, Herr JL, Imbens G (2004) Implementing matching estimators for average treatment effects in Stata. Stata J 4:290-311

American Psychiatric Association (1994) Diagnostic and statistical manual of mental disorders, 4th edn. American Psychiatric Publishing, Washington DC

American Psychiatric Association (2013) Diagnostic and statistical manual of mental disorders, 5th edn. American Psychiatric Publishing, Arlington

Anticevic A, Lisman J (2017) How can global alteration of excitation/ inhibition balance lead to the lLocal dysfunctions that underlie schizophrenia? Biol Psychiatry 81:818-820

Babiloni F, Babiloni C, Carducci F, Fattorini L, Onorati P, Urbano A (1996) Spline Laplacian estimate of EEG potentials over a realistic magnetic resonance-constructed scalp surface model. Electroencephalogr Clin Neurophysiol 98:363-373

Baddeley A (2000) The episodic buffer, a new component of working memory? Trends Cogn Sci 4:417-423

Barr MS, Rajji TK, Zomorrodi R, Radhu N, George TP, Blumberger DM, Daskalakis ZJ (2017) Impaired theta-gamma coupling during working memory performance in schizophrenia. Schizophr Res 189:104-110

Başar E (2013) Brain oscillations in neuropsychiatric disease. Dialogues Clin Neurosci 15:291-300

Başar-Eroglu C, Brand A, Hildebrandt H, Kedzior KK, Mathes B, Schmiedt C (2007) Working memory related gamma oscillations in schizophrenia patients. Int J Psychophysiol 64:39-45

Bertrand O, Tallon-Baudry C (2000) Oscillatory gamma activity in humans, a possible role for object representation. Inter J Psychophysiol 38:211-223

Buzsáki G, Wang XJ (2012) Mechanisms of gamma oscillations. Annu Rev Neurosci 35:203-225

Cowan N (2001) The magical number 4 in short-term memory, a reconsideration of mental storage capacity. Behav Brain Sci 24:87-114

Doege K, Kumar M, Bates AT, Das D, Boks MP, Liddle PF (2010) Time and frequency domain event-related electrical activity associated with response control in schizophrenia. Clin Neurophysiol 121:1760-1771

Fries P (2009) Neuronal gamma-band synchronization as a fundamental process in cortical computation. Annu Rev Neurosci 32:209-224

Fries P, Nikolić D, Singer W (2007) The gamma cycle. Trends Neurosci 30:309-316

Gruber AJ, Calhoon GG, Shusterman I, Schoenbaum G, Roesch MR, O'Donnell P (2010) More is less, a disinhibited prefrontal cortex impairs cognitive flexibility. J Neurosci 30:17102-17110

Güntekin B, Başar E (2016) Review of evoked and event-related delta responses in the human brain. Int J Psychophysiol 103:43-52

Haenschel C, Bittner RA, Waltz J, Haertling F, Wibral M, Singer W, Linden DE, Rodriguez E (2009) Cortical oscillatory activity is critical for working memory as revealed by deficits in early-onset schizophrenia. J Neurosci 29:9481-9489

Homan RW, Herman J, Purdy P (1987) Cerebral location of international 10-20 system electrode placement. Electroencephalogr Clin Neurophysiol 66:376-382

Howard MW, Rizzuto DS, Caplan JB, Madsen JR, Lisman J, Aschenbrenner-Scheibe R, Schulze-Bonhage A, Kahana MJ (2003) Gamma oscillations correlate with working memory load in humans. Cereb Cortex 13:1369-1374

Hunt MJ, Kopell NJ, Traub RD, Whittington MA (2017) Aberrant network activity in schizophrenia. Trends Neurosci 40:371-382

Ince NF, Pellizzer G, Tewfik AH, Nelson K, Leuthold A, McClannahan K, Stephane M (2009) Classification of schizophrenia 
with spectro-temporo-spatialMEG patterns in working memory. Clin Neurophysiol 120:1123-1134

Insel TR (2010) Rethinking schizophrenia. Nature 468:187-193

Jung TP, Makeig S, Humphries C, Lee TW, McKeown MJ, Iragui V, Sejnowski TJ (2000) Removing electroencephalographic artifacts by blind source separation. Psychophysiology 37:163-178

Kamiński J, Brzezicka A, Wróbel A (2011) Short-term memory capacity $(7 \pm 2)$ predicted by theta to gamma cycle length ratio. Neurobiol Learn Mem 95:19-23

Kang SS, MacDonald AW 3rd, Chafee MV, Im CH, Bernat EM, Davenport ND, Sponheim SR (2018) Abnormal cortical neural synchrony during working memory in schizophrenia. Clin Neurophysiol 129:210-221

Lakatos P, Shah AS, Knuth KH, Ulbert I, Karmos G, Schroeder CE (2005) An oscillatory hierarchy controlling neuronal excitability and stimulus processing in the auditory cortex. J Neurophysiol 94:1904-1911

Leszczyński M, Fell J, Axmacher N (2015) Rhythmic working memory activation in the human hippocampus. Cell Rep 13:1272-1282

Lett TA, Voineskos AN, Kenned JL, Levine B, Daskalakis ZJ (2014) Treating working memory deficits in schizophrenia, a review of the neurobiology. Biol Psychiatry 75:361-370

Lewis DA (2012) Cortical circuit dysfunction and cognitive deficits in schizophrenia: implications for preemptive interventions. Eur J Neurosci 35:1871-1878

Lewis DA, Fish KN, Arion D, Gonzalez-Burgos G (2011) Perisomatic inhibition and cortical circuit dysfunction in schizophrenia. Curr Opin Neurobiol 21:866-872

Lisman JE (2012) Excitation, inhibition, local oscillations, or largescale loops, what causes the symptoms of schizophrenia? Curr Opin Neurobiol 22:537-544

Lisman J, Buzsáki G (2008) A neural coding scheme formed by the combined function of gamma and theta oscillations. Schizophr Bull 34:974-980

Lisman JE, Jensen O (2013a) The $\theta-\gamma$ neural code. Neuron 2013(77):1002-1016

Lisman JE, Jensen O (2013b) The theta-gamma neural code. Neuron 77:1002-1016

Lundqvist M, Rose J, Herman P, Brincat SL, Buschman TJ, Miller EK (2016) Gamma and Beta Bursts Underlie Working Memory. Neuron 90:152-164

Mainy N, Kahane P, Minotti L, Hoffmann D, Bertrand O, Lachaux JP (2007) Neural correlates of consolidation in working memory. Hum Brain Mapp 28:183-193

Mäki-Marttunen T, Krull F, Bettella F, Hagen E, Næss S, Ness TV, Moberget T, Elvsåshagen T, Metzner C, Devor A, Edwards AG, Fyhn M, Djurovic S, Dale AM, Andreassen OA, Einevoll GT (2019) Alterations in schizophrenia-associated genes can lead to increased power in delta oscillations. Cereb Cortex 29:875-891

Missonnier P, Herrmann FR, Zanello A, Badan Bâ M, Curtis L, Canovas D, Chantraine F, Richiardi J (2012) Giannakopoulos P, Merlo MC. Event-related potentials and changes of brain rhythm oscillations during working memory activation in patients with first-episode psychosis. J Psychiatry Neurosci 37:95-105

Missonnier P, Curtis L, Ventura J, Herrmann FR, Merlo MCG (2017) Differences of temporal dynamics and signal complexity of gamma band oscillations in first-episode psychosis during a working memory task. J Neural Transm 124:853-862

Nystrom LE, Braver TS, Sabb FW, Delgado MR, Noll DC, Cohen JD (2000) Working memory for letters, shapes, and locations, fMRI evidence against stimulus-based regional organization in human prefrontal cortex. Neuroimage 11:424-446
Palva JM, Monto S, Kulashekhar S, Palva S (2010) Neuronal synchrony reveals working memory networks, predicts individual memory capacity. PNAS 107:7580-7585

Park YU, Jeong J, Lee H, Mun JY, Kim JH, Lee JS, Nguyen MD, Han SS, Suh PG, Park SK (2010) Disrupted-in-schizophrenia 1 (DISC1) plays essential roles in mitochondria in collaboration with Mitofilin. Proc Natl Acad Sci USA 107:17785-17790

Perrin F, Bertrand O, Pernier J (1987) Scalp current density mapping, value and estimation from potential data. IEEE Trans Biomed Eng 34:283-288

Pratt J, Dawson N, Morris BJ, Grent-'t-Jong T, Roux F, Uhlhaas PJ (2017) Thalamo-cortical communication, glutamatergic neurotransmission and neural oscillations: a unique window into the origins of ScZ? Schizophr Res 180:4-12

Prochiantz A (2014) Processus morphogénétiques. Annuaire du Collège de France 2012-2013. Sci Math Phys Nat 113:331-353

Puvvada KC, Summerfel A, Du X, Krishna N, Kochunov P, Rowland LM, Simon JZ, Hong LE (2018) Delta vs gamma auditory steady state synchrony in schizophrenia. Schizophr Bull 44:378-387

Rajji TK, Zomorrodi R, Barr MS, Blumberger DM, Mulsant BH, Daskalakis ZJ (2017) Ordering information in working memory and modulation of gamma by theta oscillations in humans. Cereb Cortex 27:1482-1490

Reilly TJ, Nottage JF, Studerus E, Rutigliano G, Micheli AI, FusarPoli P, McGuire P (2018) Gamma band oscillations in the early phase of psychosis: a systematic review. Neurosci Biobehav Rev 90:381-399

Roux F, Uhlhaas PJ (2014) Working memory and neural oscillations, $\alpha-\gamma$ versus $\theta-\gamma$ codes for distinct WM information? Trends Cogn Sci 18:16-25

Schoffelen JM, Oostenveld R, Fries P (2005) Neuronal coherence as a mechanism of effective corticospinal interaction. Science 308:111-113

Senkowski D, Gallinat J (2015) Dysfunctional prefrontal gammaband oscillations reflect working memory and other cognitive deficits in schizophrenia. Biol Psychiatry 77:1010-1019

Spencer KM, Nestor PG, Perlmutter R, Niznikiewicz MA, Klump MC, Frumin M, Shenton ME, McCarley RW (2004) Neural synchrony indexes disordered perception and cognition in schizophrenia. Proc Natl Acad Sci USA 101:17288-17293

Spencer KM, Niznikiewicz MA, Shenton ME, McCarle RW (2008) Sensory-evoked gamma oscillations in chronic schizophrenia. Biol Psychiatry 63:744-747

Steriade M (2006) Grouping of brain rhythms in corticothalamic systems. Neuroscience 137:1087-1106

Uhlhaas PJ, Singer W (2010) Abnormal neural oscillations and synchrony in schizophrenia. Nat Rev Neurosci 11:100-113

Uhlhaas PJ, Singer W (2013) High-frequency oscillations and the neurobiology of schizophrenia. Dialogues Clin Neurosci 15:301-313

Uhlhaas PJ, Haenschel C, Nikolić D, Singer W (2008) The role of oscillations and synchrony in cortical networks and their putative relevance for the pathophysiology of schizophrenia. Schizophr Bul 5:927-943

Uhlhaas PJ, Roux F, Rodriguez E, Rotarska-Jagiela A, Singer W (2010) Neural synchrony and the development of cortical networks. Trends Cogn Sci 14:72-80

Ventura J, Green MF, Shaner A, Liberman RP (1993) Training and quality assurance with the brief psychiatric rating-scale - the drift busters. Int J Methods Psychiatr Res 3:221-244

Ward M (2011) The thalamic dynamic core theory of conscious experience. Conscious Cogn 20:464-486

Zaitsev AV, Lewis DA (2013) Functional properties and short-term dynamics of unidirectional and reciprocal synaptic connections 
between layer $2 / 3$ pyramidal cells and fast-spiking interneurons in juvenile rat prefrontal cortex. Eur J Neurosci 38:2988-2998

Zhang Y, Llinas RR, Lisman JE (2009) Inhibition of NMDARs in the nucleus reticularis of the thalamus produces delta frequency bursting. Front Neural Circuits 3:20
Publisher's Note Springer Nature remains neutral with regard to jurisdictional claims in published maps and institutional affiliations. 